\title{
Pigmented tumor in the nostril
}

\author{
Nawel Jaada, M.D. ${ }^{1}$, Ines Zaraa, M.D. ${ }^{1}$, Ines Chelly, M.D. ${ }^{2}$, Rym Cheikhrouhou, M.D. ${ }^{1}$, \\ Sondes Trojjet, M.D. ${ }^{1}$, Dalenda El Euch, M.D. ${ }^{1}$, Mourad Mokni, M.D. ${ }^{1}$, Slim Haouet, M.D. ${ }^{2}$, \\ Amel Ben Osman, M.D. ${ }^{1}$
}

${ }^{1}$ Dermatology Department, La Rabta Hospital, Tunis, Tunisia

${ }^{2}$ Pathology Department, La Rabta Hospital, Tunis, Tunisia

Key words: inverted follicular keratosis, seborrheic keratosis

Citation: Jaada N, Zaraa I, Chelly I, et al. Pigmented tumor in the nostril. Dermatol Pract Concept 2011;1(1):11. http://dx.doi.org/10.5826/ dpc.0101a11.

Editor: Harald Kittler, M.D.

Received: October 1, 2010; Accepted: January 27, 2011; Published: October 31, 2011

Copyright: @2011 Jaada et al. This is an open-access article distributed under the terms of the Creative Commons Attribution License, which permits unrestricted use, distribution, and reproduction in any medium, provided the original author and source are credited.

Funding: None.

Competing interests: The authors have no conflicts of interest to disclose.

All authors have contributed significantly to this publication.

Corresponding author: Ines Zaraa, M.D., Assistant Professor, Dermatology Department, La Rabta Hospital, Jabbari, Bab Saadoun, Tunis, 1007 Tunisia. Tel: +21698307425, Fax: +21671569449. E-mail: inesrania@myway.com.

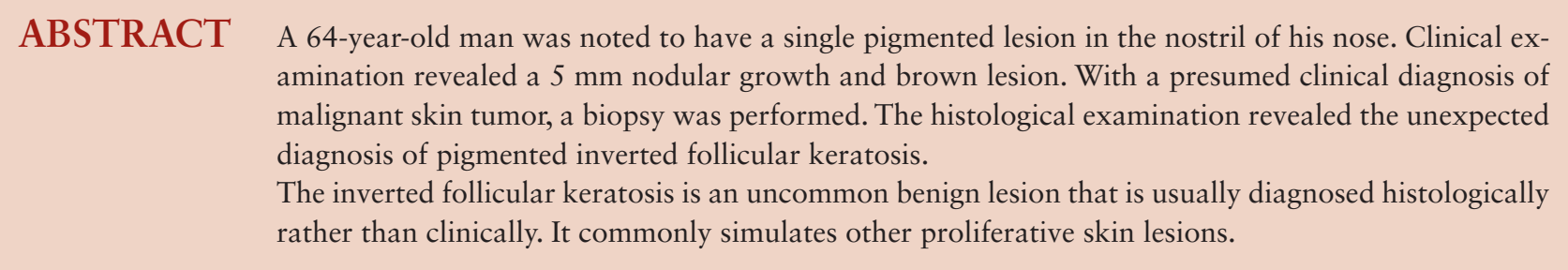

\section{Introduction}

The inverted follicular keratosis is an uncommon benign lesion that is usually diagnosed histologically rather than clinically. We report on a singular case of a pigmented skin lesion, which was revealed on histological examination to be a pigmented inverted follicular keratosis.

\section{Observation}

We present a case of a white 64-year-old man, with no significant co-morbidities, who had a remote history of a pro-

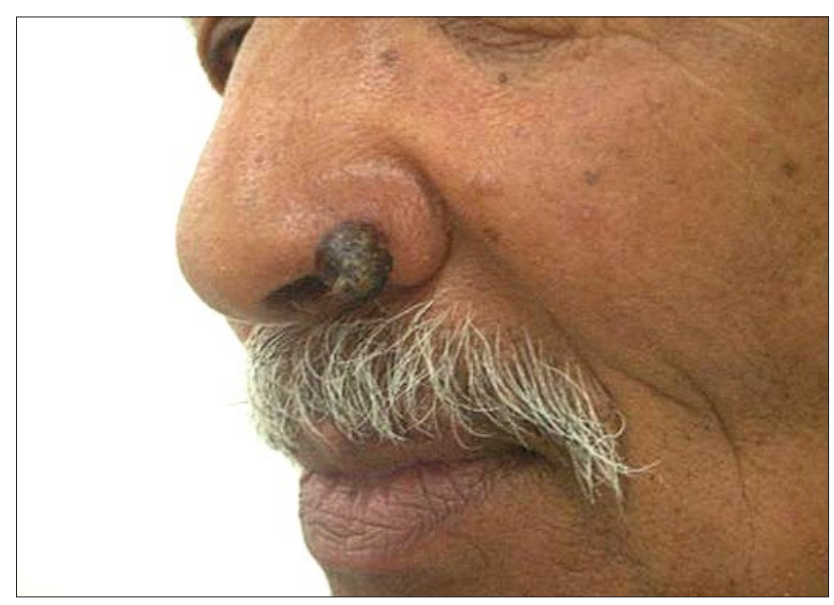

Figure 1. Exophytic, corneal brown lesion located in the left nostril. 

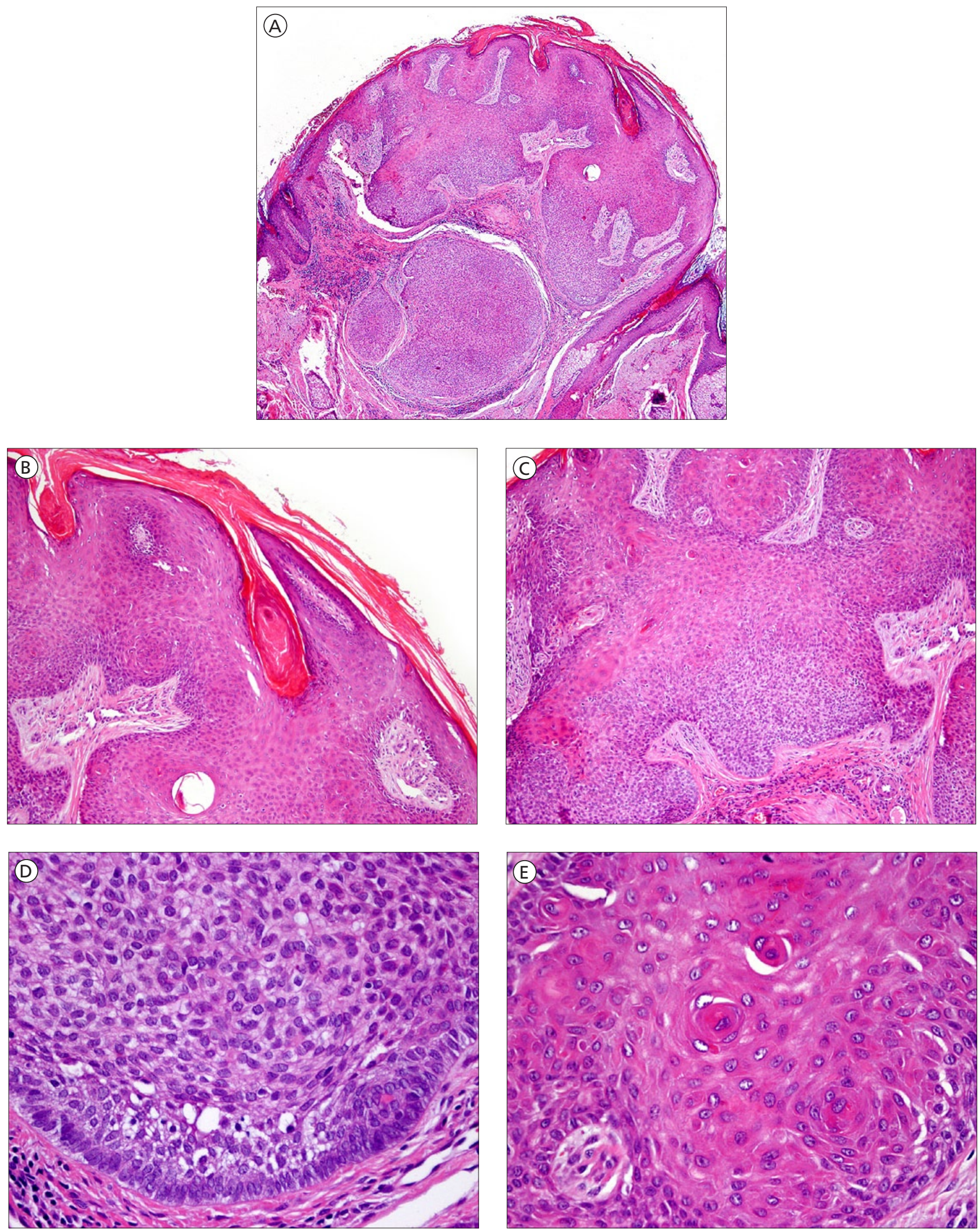

Figure 2. A: Epithelial proliferation both above and below the level of residual epidermis (hematoxylin and eosin (H\&E) stain); B: Keratin crypt formation at the surface of the lesion with hyperkeratotic epithelium and acanthosis (H\&E stain); C: Epithelial proliferation containing a squamous eddy formation (H\&E stain); D: High-power photomicrograph depicting base of inverted follicular keratosis with peripheral palisading of basaloid cells at the periphery of the tumor lobules and intercellular edema; E: Squamous eddy formation. 
longed sun exposure. The patient presented with an asymptomatic nodular growth and brown lesion measuring $5 \mathrm{~mm}$ in diameter and located in the left nostril of the nose (Figure 1); the patient had noticed the appearance of the lesion a few months previous to his visit to us. Clinically, the tumor appeared to be limited to the skin with no evidence of deep infiltration. Basal cell carcinoma, squamous cell carcinoma, melanoma and seborrheic keratosis were considered as possible diagnoses.

The patient had a skin quarter biopsy. The histopathologic findings, illustrated in Figures 2a-e show downward growth of the epithelium with intralesional corneal cystic inclusions, surrounded by nonspecific chronic inflammatory infiltration, with no evidence of cellular atypia. The epidermis revealed hyperkeratosis, acanthosis, and papillomatosis with a sharp dermarcation line. Polymerase chain reaction (PCR) for human papillomavirus (HPV) was negative. Four months later, the patient reported a spontaneous regression of the remaining tumor (Figure 3).

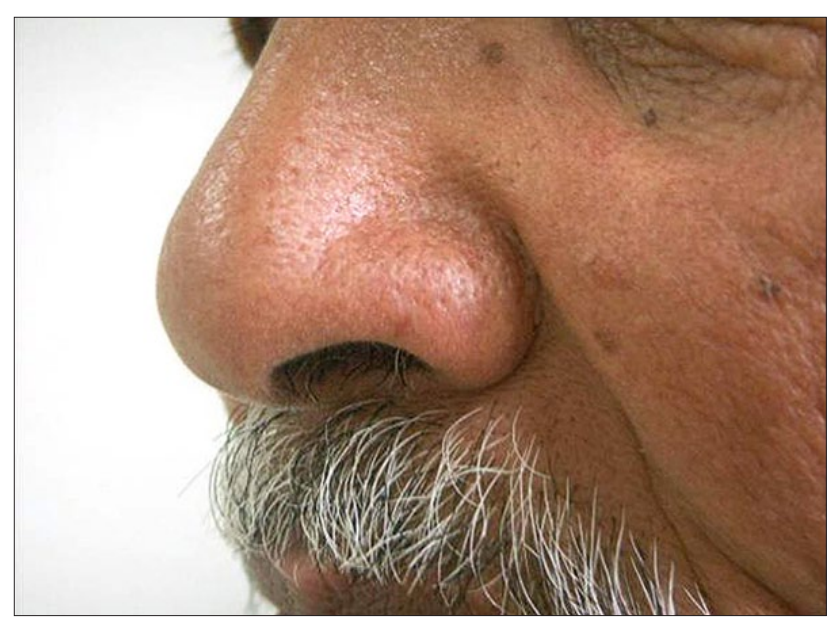

Figure 3. Spontaneous regression of the inverted follicular keratosis

\section{Comment}

Histologic examination of this lesion revealed features consistent with inverted follicular keratosis (IFK). A mild controversy currently exists in the literature concerning the origin of IFK. Helwig and others [1,2] support the hair follicle as the origin of this lesion, while some authors believe that this lesion is best characterized as a variant of seborrheic keratosis (SK) that has been irritated, [3] and others consider it as distinct entity [4]. It is characterized by a squamous epithelial expansion of the infundibular portion of the hair follicle in an exophytic and endophytic pattern.

IFK is a benign skin lesion reported initially by Helwig [1] that occurs in middle aged or older patients ( $>50$ years), with median age at presentation of 69 years. Men are affected about twice as often as women. In IFK the lesions are generally asymptomatic, firm, and pinkish papules, but it can present chromatic variants, typically from yellow to brown, in relation to the content of melanin [5]. The lesion most often arises as a solitary skin nodule on the face (85\%) [4]; however, multiple IFK have been described in Cowden's syndrome [6]. The cheek and the upper lip are the sites of predilection; other sites affected are the chin, forehead, eyebrow, nose, and eyelid [7]. Most of the lesions are between 3 and $8 \mathrm{~mm}$ in maximum diameter, but a few reach a size of $10 \mathrm{~mm}$. The duration of the lesion varies between six weeks and three years in most cases, and in some cases as long as 15 years has been reported [7].

IFK can be misdiagnosed clinically as different skin cancers. Indeed, in our observation the lesion mimicked the clinical appearance of basal cell carcinoma, or pigmented SK, or benign adnexal tumor. The diagnosis was based on histological examination, therefore, and a skin biopsy should always be performed in order to exclude its malignant nature. In fact, IFK can also be mistaken clinically as melanoma because of the heavily pigmented nature of the lesion [8].

In our observation, the histological appearance of the tumor fits perfectly with the description in the literature. Histopathological examination showed epithelial proliferation above and below the level of the epidermis with hyperkeratosis, parakeratosis and papillomatosis of the epidermis. In addition, we note a broad fingerlike downward epithelial proliferation containing occasional horn cysts, which consists of two cell types, basal cells located in the periphery and malpighian cells in the center. These structures are named "squamous eddies." Generally, the base of the lesion is sharply delineated from the underlying corium and contains intercellular edema and a minimal inflammatory infiltrate with no evidence of cellular atypia. All these morphological findings are suggestive of IFK.

Irritated seborrheic keratosis is the first histopathological differential diagnosis. Acceptance of the "irritation theory" implies that a lesion of seborrheic keratosis must be present before the irritation can occur. Since a primary characteristic of seborrheic keratosis is its lack of penetration into the dermis, the presence of a prominent downward growth pattern in IFK suggests a different theory of origin. The presence of surface crypts, horn cysts, a downward growth pattern, and frequent association with the hair follicle in IFK favors a follicular origin. Some authors considered IFK as distinct from SK [4]. In addition to exophytic and endophytic patterns, a few koilocytes may occur in IFK and never in SK. Appearance of eosinophilic cytoplasm in IFK contrast with the appearance of basaloid cell cytoplasm in SK. Lastly, the squamous eddies are not pathognomonic of the IFK, but they are particularly numerous [9].

In our case the only close differential diagnosis is trichilemmoma. IFK shares some histologic features with trichil- 
emmona. There is a proliferation of pale-staining outer root sheath-type epithelium that is well circumscribed and surrounded by a fibrotic stroma. Both lesions show peripheral palisading of basaloid cells at the periphery of the tumor lobules. However in IFK, there are concentric layers of squamous cells with evidence of keratinization forming squamous eddies. In addition, some authors postulate that HPV is associated to trichilemmona lesions [10]. The PCR for HPV performed in our case was negative.

The histopathological diagnosis of IFK can mimic also verruca vulgaris, viral warts, and squamous cell carcinoma. IFKs can contain cellular atypia, and squamous eddies bear some resemblance to those of squamous cell carcinoma $[11,12]$. The well-delineated base, broad acanthotic epithelial downgrowth, squamous eddy formation, and lack of epithelial dysplasia support a diagnosis of IFK here [2]. The pathologist should be most attentive whenever histopathologic features suggestive of IFK are present. Indeed, more than half of these lesions have been mistaken for squamous cell carcinoma on initial evaluation, and this can result in unnecessarily drastic treatment of a benign lesion.

\section{References}

1. Helwig EB. Inverted follicular keratosis. In: Seminar on the Skin: Neoplasms and Dermatosis. Washington, DC, American Society of Clinical Pathologists, International Congress of Clinical Pathology, 1954.

2. Adrian J. Inverted follicular keratosis of the lip. Oral Surg Oral Med Oral Pathol 1984;57(6):625-30.

3. Lever WF. Inverted follicular keratosis is an irritated seborrheic keratosis. Am J Dermatopathol 1983;5(5):474.

4. Azzopardi JG, Laurini R. Inverted follicular keratosis. J Clin Pathol 1975;28(6):465-71.

5. Villaret AB, Gily B, Aga A. Inverted follicular keratosis of the nasal vestibule. Otolaryngol Head Neck Surg 2009;141(2):288-9.

6. Ruhoy SM, Thomas D, Nuovo GJ. Multiple inverted follicular keratoses as a presenting sign of Cowden's syndrome: case report with human papillomavirus studies. J Am Acad Dermatol 2004;51(3):411-5.

7. Soylu L, Akcali C, Aydogan LB, Ozsahinoglu C, Tuncer I. Inverted follicular keratosis. Am J Otolaryngol 1993;14(4):247-8.

8. Thom GA, Quirk CJ, Heenan PJ. Inverted follicular keratosis simulating malignant melanoma. Australas J Dermatol 2004;45(1):55-7.

9. Choi HJ, Yun SK, Kim HU, Ihm CW. Squamous eddies in irritated seborrheic keratosis. Am J Dermatopathol 2007;29(1):28-31.

10. Stierman S, Chen S, Nuovo G, Thomas J. Detection of human papillomavirus infection in trichilemmomas and verrucae using in situ hybridization. J Cutan Pathol 2010;37(1):75-80.

11. Shih CC, Yu HS, Tung YC, Tsai KB, Cheng ST. Inverted follicular keratosis. Kaohsiung J Med Sci 2001;17(1): 50-4.

12. Roth LM, Look KY. Inverted follicular keratosis of the vulvar skin: a lesion that can be confused with squamous cell carcinoma. Int J Gynecol Pathol 2000;19(4):369-73. 